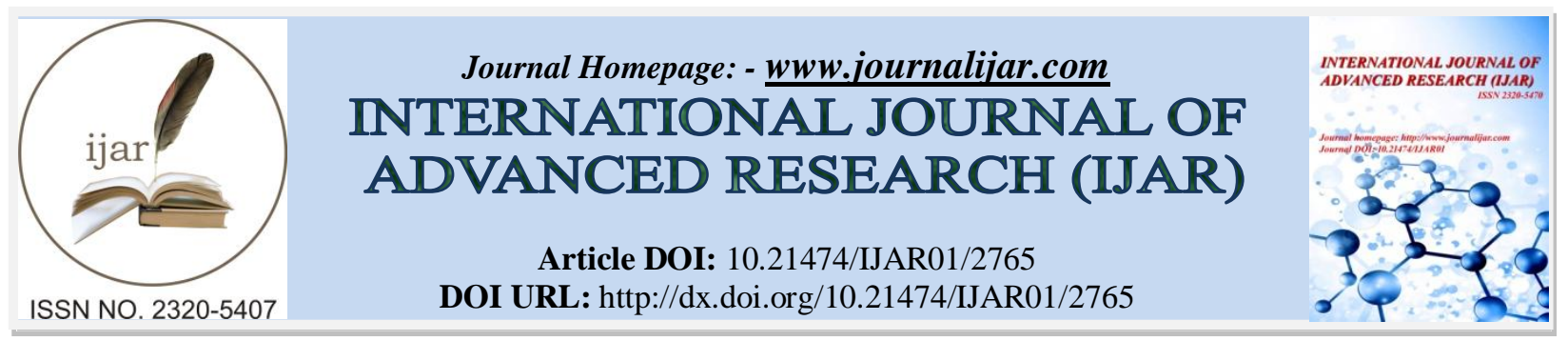

RESEARCH ARTICLE

\title{
MENTAL STATES IN WOMEN: THE ROLE OF FAMILY
}

Eshrat Ara.

Research Scholar, Department of Psychology ,University of Kashmir, Hazratbal, Srinagar, 190006, J\&K, India.

\section{Manuscript Info}

Manuscript History

Received: 15 November 2016

Final Accepted: 17 December 2016

Published: January 2017

Key words:-

family functioning; positive mental

states; negative mental states.

\section{Abstract}

Background: Women disproportionately suffer from mental health disorders and are more frequently subject to social causes. Most findings implicate negative mental states are the offshoots of inadequate interaction with the environment and family environment is the chief cause. This study extends existing research in the field of mental health that focuses on the effects of family functioning on positive and negative mental states in females.

Aims: This study tested the hypothesized model that healthy family functioning leads to increased positive mental states and, subsequently, reduced negative mental states in females.

Methods: A sample of undergraduate and postgraduate $(n=201)$ female students completed survey packets in order to test this sequence of relationships on the General Family Functioning Scale, Oxford Happiness Questionnaire, Herth Hope Index, Life Orientation Test and Depression Anxiety Stress Scale.

Statistical analysis used: A Structural Equation Model was then constructed and evaluated based on the tested measurement model of latent factors.

Results: The model yielded significant effect. The findings from the study indicated that healthy family functioning and positive mental states are positively related to each other, but negatively related to negative mental states. The findings provide compelling empirical evidence that healthy family functioning reduce the negative mental states by enhancing the positive mental resources. Thus negative mental states can be accurately modeled as an outcome of family functioning mediated by positive mental states.

Conclusions: This study suggests that combined interventions, targeting family functioning and increasing positive mental states may be particularly useful in addressing negative mental states in females. The research contributes recommendations for policy, practice and further studies.

Copy Right, IJAR, 2016,. All rights reserved.

\section{Introduction:-}

Women's health within the policy domain is often defined as reproductive and identified with women's children's health. Although, this perspective is well-founded given that the health of women is well documented to have a positive impact on the general health of all members of a society (Marcia, 1997; Ware \& Good, 1995). But, the 
question rises, what about programs designed to address women's needs as women? Such question challenges the traditional health policies and programs and calls for definitions of women's health which are broader than the reproductive and the maternal, incorporating mental health as well (Rosenfield \& Maine, 1985). Women's wellbeing is not solely determined by biological factors and reproduction, but also by psychological factors. So the need is to mainstream mental health issues as well, because women disproportionately suffer from mental health disorders and are more frequently subject to social causes that lead to mental distress (Van der Kwaak, Vanden Engel, \& Richters, 1991).

\section{Previous research on mental states:-}

Comparative analysis of empirical studies of mental disorders reveals a consistency across diverse societies and social contexts, revealing negative mental states more prevalent among women which can be related to the social circumstances of many women's lives (Ware \& Good, 1995). Most findings implicate negative mental states are the offshoots of inadequate interaction with the environment and family environment is the chief cause (Chauhan, 2006; Chorpita \& Barlow, 1998; Das, 1994; Joshi \& Tomar, 2006; Parker, 1983; Sharma, Verma \& Malhotra, 2008). Links between emotional deprivation or affectionless control, and psychological distress in women have also been documented in many studies, including work carried out in Brazil, Mexico and Pakistan (kaya, 1985; Malik, Bukhtiari, \& Good, 1992; Naeem, 1992; Nancy, 1995; Nations \& Rebhun, 1988). Chorpita and Barlow (1998) articulated a model of the environmental influences on the development of negative mental states. Evidence from a variety of sources suggests that life experience with diminished control may foster a cognitive style characterized by an increased probability of interpreting or processing subsequent events as out of one's control, which may represent a psychological vulnerability for mental distress.

Psychologists have discovered that there is a set of human strengths that are the most likely buffer against mental illness: happiness, courage, optimism, interpersonal skill, hope, honesty and perseverance (Khordzanganeh, Heidarie, \& Naderi, 2014; Seligman, 1998). A number of positive predictors of happiness have been identified, including satisfaction with relationships with people from whom support has been received (Lu \& Argyle, 1992). This speaks to the importance of positive relationships with others for the well-being of an individual. Research suggests that hope and optimism are beneficial for physical and psychological well-being (Aspinwall \& Taylor; 1992; Scheier, Carver, \& Bridges, 1994). Carr (2004) contended that positive mental states are positively correlated with, and are predictive of, physical and mental health. Seligman and Csikszenthihalyi (2000) called for counselors and psychologists working with families and other settings to develop climates that foster these strengths.

\section{Family Functioning:-}

From the work of Ackerman (1959), Jackson (1965), Minuchin (1974), and Bowen (1978) came family systems theory, developed on the basis of general systems theory (Bertalanffy, 1973). General systems theory includes basic ideas from Gestalt psychology, focusing on the whole, and Kert Lewin's field theory (1951) (Schwab, Stephenson, $\&$ Ice, 1993). A primary concept in the family systems theory is that the family includes interconnected members, and each member influences the other in predictable and recurring ways (Fingerman \& Bermann, 2000; Van Velsor \& Cox, 2000). Family functioning is more related to the transactional and systematic properties of the family system than to the intrapsychic characteristics of the individual (Westley \& Epstein, 1969). Family functioning refers to set of basic attributes about the family system that characterize and explain how a family system operates (McCubbin \& Thompson, 1991). Family therapists and theorists have identified different attributes of family functioning. McMaster's Model of Family Functioning (Epstein, Bishop, \& Levin, 1978) conceptualizes family functioning as comprising of six dimensions: problem solving, communication, roles, affective responsiveness, affective involvement, and behavioural control. McMaster Model of Family Functioning (MMFF) (Epstein et al., 1978) is a clinically orientated conceptualization of families. It describes the structural and organizational properties of the family group and the patterns of transactions among family members which have been found to distinguish between healthy and unhealthy families. Many studies have examined family functioning of patients with psychiatric disorders, including depression, adjustment disorders, anxiety disorder, etc. (Friedmann, McDermut, Solomon, Ryan, keitner, \& Miller, 1997; Keitner, Miller, \& Ryan, 1993; Miller, Keitner, whisman, Ryan, Epstein, \& Bishop, 1992).

\section{Contributions and Limitations of previous research:-}

Despite the importance of research investigating mental states in women, several key limitations of the research exist. These limitations are detailed below. In detailing these limitations, however, we also recognize the contribution of previous studies, and indicate how these contributions relate to the limitations identified. 
Positive mental states have usually been shown to be related with negative mental states in research studies because positive mental states are assumed to be psychological beliefs which are known to be protective of mental health (Taylor, Kemeny, Reed, Bower, \& Gruenewald, 2000). The elements in the environment are also considered to support the development and maintenance of mental health (Seligman, 2002). Research studies have portrayed unhealthy family environment as a precursor of mental distress. Further it has been shown that healthy family functioning is supportive of development of positive beliefs, thoughts and behaviours.

Most of the research studies relating family functioning and mental problems have been conducted on adolescents. When studies have focused on mental states as a dependent variable, they have (like the present study) often focused on reducing negative mental states. However, in doing so, previous studies have adopted the general approach identifying antecedent social or psychological variables to mental states. However, these studies often investigate these variables in a general or theoretical sense, without direct psychological measurement or modeling, and/or using weaker methodologies. This study, in contrast, examines antecedent social and psychological variables of mental states by directly modeling negative mental states as the casual outcome of a network of these variables. In this way, this study follows the more rigorous approach.

\section{The present study:-}

For the reasons above, we sought to investigate mental states: (a) as a dependent variable, (b) using sophisticated latent variable modeling approaches, (c) with a focus on identifying a network of interrelated antecedent variables that may reduce negative mental states and (d) taking gender specific sample of women, (e) in a non-western context. More specifically, we propose to test a model hypothesizing that healthy family functioning leads to increased positive mental states and, subsequently, reduced negative mental states, i.e.,

Healthy Family Functioning $\longrightarrow$ Increased Positive Mental States

The structural model tested in this research implies that healthy family functioning leads to increased resources of positive mental states. In turn, positive mental states have the strength to provide a cognitive and affective defense against negative mental states. Importantly, we hypothesize that the direct effects of family functioning on negative mental states will be less important than the indirect effects of family functioning acting on negative mental states through positive mental states. It is assumed that when healthy family environment leads to increased positive mental states then negative mental states are potentially reduced significantly because positive mental states implies resources of strength which are expected to counteract the negative mental states.

In this study the antecedent variable family functioning is defined as measured by General Family Functioning Scale, an overall measurement of family functioning, one of the subscales of Family Assessment Devise (FAD), developed on the basis of McMaster's Model of Family Functioning (Epstein, et al., 1978). The mediating variables in model is positive metal states defined as happiness, as measured by Oxford happiness Questionnaire; hope, as measured by Herth Hope Index; and optimism, as measured by Life Orientation Test - Revised. And the causal variable in the model i.e., negative mental states is defined as depression, anxiety, and stress, as measured by Depression anxiety Stress Scale.

\section{Purpose of the study:-}

The specific purpose of the study was to test the veracity of a model hypothesizing mental states to be the consequence of family. In order to achieve this purpose the measures in the study were first tested in a measurement model. Based on the measurement model, a Structural Equation Model (SEM) of Negative Mental States with family functioning and positive mental states as antecedent variables was then constructed and evaluated.

For the purposes of operationalizing our SEM model, we accepted the validity of certain relationships between constructs of interest already postulated in the literature, but noted that these relationships have not been tested within a causal modeling framework. In other words, for the purpose of constructing testable causal and measurement models, we assumed that the postulated relationships drawn from the literature, and operationalized in the models tested in this research, were at least plausible. At the same time, for the purpose of actually testing the models, we did not automatically assume that the postulated relationships operationalized in the models would hold in any particular sample/population. The study, then, both accepts the validity of the postulated relationships (for 
model construction purposes), and does not (automatically) accept the validity of the postulated relationships (for model testing purposes).

\section{Method:-}

\section{Participants:-}

The study was conducted in the Kashmir valley of India. Data were collected from 201 undergraduate and postgraduate female students, studying in University of Kashmir, Srinagar. They ranged in age from 18 to 30 years, with the average age of 23 years.

\section{Instruments:-}

The present study utilized self-reported instruments which are as follows.

a. McMaster General Family Functioning Scale is a 12-item (6 phrased positively and other 6 negatively) subscale of McMaster Family Assessment Device (FAD) developed by Epstein, Baldwin, \& Bishop (1983), on the basis of the McMaster Model of Family Functioning (MMFF; Epstein, et al., 1978; Westley \& Epstein, 1969). General functioning scale assesses the overall health/pathology of the family. Scores range from 1 (strongly agree) to 4 (strongly disagree).

b. Life Orientation Test - Revised (LOT-R; Scheier, Carver, \& Bridges, 1994) consists of 10 items, 3 items measure optimism, 3 items measure pessimism, and 4 items serve as fillers. It has a 5 -point scale ranging from 0 (strongly disagree) to 4 (strongly agree). Items 3, 7, and 9 are reverse scored. Items 2, 5, 6 and 8 are fillers and are not scored.

c. Herth Hope Index (HHI; Herth, 1992), an abbreviated version of Herth Hope Scale, consists of 12 items that are based on three dimension, namely, temporality and future, positive readiness and expectancy, and interconnectedness. It is a 4 point scale, response ranging from1 (strongly disagree) to 4 (strongly agree). Items 3 and 6 are reverse scored.

d. Oxford Happiness Questionnaire (OHQ; Argyle \& Hills, 2002), has been derived from the Oxford Happiness Inventory (OHI). The OHQ short scale includes eight items of the OHI, (some are phrased positively and others negatively) which can be endorsed on a six-point Likert scale, ranging from "strongly agree (6)" to "strongly disagree (1)'. Items 1, 4, and 8 are reverse scored.

e. Depression Anxiety Stress Scale (DASS-21) has been developed by Lovibond and Lovibond, 1995. It consists of 3 subscales of 7 items each: Depression (assesses dysphoria, hopelessness, devaluation of life, selfdeprecation, lack of interest/involvement, anhedonia and inertia); Anxiety (assesses autonomic arousal, skeletal muscle effects, situation anxiety and subjective experience of anxious effect); and Stress (assesses levels of nonchronic arousal through difficulty relaxing, nervous arousal and being easily upset/agitated, irritable/overreactive and impatient). The rating scale ranges from 0 (never) to 3 (almost always).

\section{Procedure:-}

Participants were asked to complete a survey package consisting of all the instruments described above with proper instructions. The questionnaires were distributed to the participants in the different departments and collected on the subsequent days

\section{Data analysis:-}

A measurement model of three latent variables (LVs) was created corresponding to family functioning, positive mental states, and negative mental states, and the strength of the relationships between the three LVs was the subject of investigation. Confirmatory factor analysis (CFA), using Analysis of Moment Structures (AMOS 20.0), evaluated the adequacy of the measurement model, prior to testing more complex SEM. The key interest of the present study was the relationships between family functioning, positive mental states and negative mental states. In this study, Hierarchical Structural Equation Modeling (HSEM - Byrne, 1998; Kaplan, 2000) was used to test these relationships.

Structural equation modeling (SEM), conducted with AMOS 20.0, was used to test the study hypotheses. SEM was used because it corrects for measurement error and can estimate both direct and indirect (mediated) effects simultaneously. Constructs were represented with item-parcels (i.e., sums of items) (Bandalos \& Finney, 2001). The use of parcels is considered acceptable as long as the indicators represent a single dimension and are closely related to one another (Little, Cunningham, Shahar, \& Widaman, 2002). 


\section{Model fit:-}

Model fit in CFA and SEM Models may be assessed through a combination of parameter investigations (all parameters should be within acceptable values), the chi-square/ degrees of freedom ratio (which, ideally, should be close to, or less than, two), and various relative fit indices (which measure model fit relative to a "baseline" model hypothesizing a unidimensional factor structure) and information criteria, which provide a numerical estimate of the explanatory power of model based on certain assumptions about, and characteristics of, the model (see $\mathrm{Hu} \&$ Bentler, 1999). In this study, we used standard indices and cut-off values to evaluate fit: the Root Means Square Error of Approximations (RMSEA < .08), and the Goodness-of-Fit Index (GFI > .90) and Comparative Fit Index (CFI > .90) (see Kline, 1998), as measures of model fit, with all parameters estimated using the maximum likelihood procedure.

\section{Results:-}

\section{Confirmatory factor analyses:-}

A correlation matrix was computed among the family functioning, positive mental states, and negative mental states variable domains (see Table 1). With three exceptions in the, all intra-domain correlations were found significant, demonstrating overall medium to high size relationship (Cohen, 1988).

CFA was conducted on the overall measurement model, including all three latent variables, and it produced satisfactory model fit, $\mathrm{X}^{2}(17, \mathrm{~N}=201)=53.58, \mathrm{p}<.001($ ratio $=3.15)$; CFI $=.93$; GFI $=.94$; RMSEA $\left.=.10\right)$, indicating that the observed variables were good indicators of the latent variables and the three latent variables represented separate constructs. Fig. 1 indicates that the indicators displayed high factor loadings. The average factor loading for all indicators was 0.71 (approx.).

Table 2 indicates that mental states (positive and negative) factors are negatively and statistically significantly related to each other. Family functioning factor is positively and statistically significantly related to positive mental states, but negatively related to negative mental states. This pattern of relationships is precisely as predicted theoretically.

\section{Structural Equation Modeling (SEM):-}

To test of the relationship between the predictor latent factor, that is, healthy family functioning and outcome latent factors of positive and negative mental states, a SEM was applied. Table 3 indicates that the model fit the data well. The chi-square/degrees of freedom ratio was close to two for the SEM model, GFI $=.96, \mathrm{CFI}=.96$, and the RMSEA $=.07$, less than 0.08 .

Fig. 1 indicates that all relationships between the latent variables were as predicted. Healthy family functioning is significantly and positively related to positive mental states, which is significantly and negatively related to negative mental. Thus, positive mental states act as mediating variable between healthy family functioning and negative mental states in women. The substantially good fit of all models allows for valid inferences to be drawn from the models. These inferences include that: (a) The survey scales represent substantially good measures of the factors they are intended to measure; (b) Negative mental states can be accurately modeled as an outcome of family functioning mediated by positive mental states.

Table 1:Correlations among Family Functioning, Positive and Negative Mental States Indicators

\begin{tabular}{|c|c|c|c|c|c|c|c|}
\hline Variable & 2 & 3 & 4 & 5 & 6 & 7 & 8 \\
\hline \multicolumn{8}{|c|}{ Family Functioning Domain } \\
\hline Negative & $-.54 * * *$ & $-.28 * * *$ & $-.36^{* * *}$ & $-.34 * * *$ & $.45 * * *$ & $.25 * * *$ & $.34 * * *$ \\
\hline Positive & - & $.27 * * *$ & $.37 * * *$ & $.23 * *$ & $-.24 * *$ & -.08 & -.11 \\
\hline \multicolumn{8}{|c|}{ Positive Mental States Domain } \\
\hline Happiness & & - & $.59 * * *$ & $.43 * * *$ & $-.44 * * *$ & $-.20 * *$ & $-.17 *$ \\
\hline Hope & & & - & $.53 * * *$ & $-.48 * * *$ & -.14 & $-.25 * * *$ \\
\hline Optimism & & & & - & $-.44 * * *$ & $-.19 * *$ & $-.18 *$ \\
\hline \multicolumn{8}{|c|}{ Negative Mental States Domain } \\
\hline Depression & & & & & - & $.49 * * *$ & $.51 * * *$ \\
\hline Anxiety & & & & & & - & $.52 * * *$ \\
\hline Stress & & & & & & & - \\
\hline
\end{tabular}

$* p<.05 . * * p<.01 . * * * p<.001$. 
Table 2:- Matrix outlining Factor Correlation

\begin{tabular}{|l|c|c|c|}
\hline & Family Functioning & Positive Mental States & $\begin{array}{c}\text { Negative Mental } \\
\text { States }\end{array}$ \\
\hline Family Functioning & - & $.51^{*}$ & $-.55^{*}$ \\
\hline Positive Mental States & & - & $-.64^{*}$ \\
\hline Negative Mental States & & & - \\
\hline
\end{tabular}

$$
* p<0.001 \text {. }
$$

Table 3:- Model Fit Statistics

\begin{tabular}{|l|c|c|c|c|c|c|c|}
\hline Model & Description & $x 2$ & df & $x 2 /$ df & GFI & CFI & RMSEA \\
\hline M1 & $\begin{array}{c}\text { First-order model: } \\
8 \text { indicators, 3 factors }\end{array}$ & 53.58 & 17 & 3.15 & .94 & .93 & .10 \\
\hline M2 & SEM model for Mental states & 35.75 & 17 & 2.10 & .96 & .96 & .07 \\
\hline
\end{tabular}

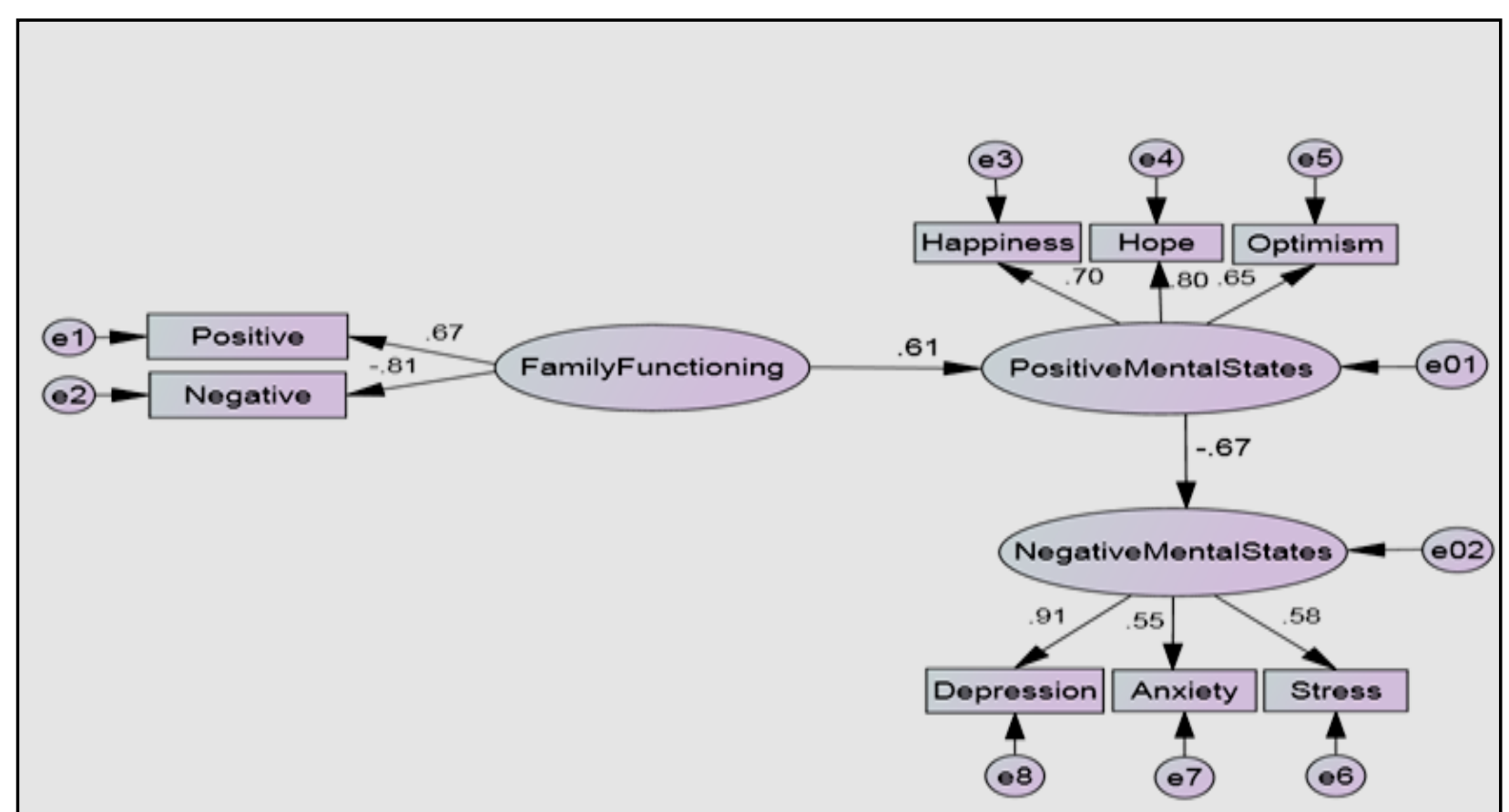

Figure 1. SEM Model of Family Functioning and Mental States 


\section{Discussion:-}

The findings from the study indicate that healthy family functioning and positive mental states are positively and statistically significantly related to each other, but negatively related to negative mental states. This pattern of relationships is consistent with the findings of previous research studies conducted in both western and non-western contexts.

However, these variables have not been extensively modeled together as interacting variables influencing important outcomes. In this study we demonstrate that positive mental states mediates the relationship between family functioning and negative mental states - thus clarifying the way these two variables may work together to influence outcome, i.e., healthy family functioning leads to increased positive mental states and reduced negative mental states.

Theoretically, then, the observed relationships support our model which suggests that healthy family functioning ought to increase positive mental states, but with positive mental states having the larger direct impact on negative mental states. Put another way, our underlying hypothesis is that family environment is necessary for a reduction in negative mental states not because they directly impact on negative mental states, but because they directly enhance positive beliefs and thoughts, with the latter resulting in a direct reduction in negative mental states.

Earlier studies have typically explored the relationship of the variables with adolescents. In this study we examined this relationship in female students at the university level, and demonstrated that positive mental states is an important mediating variable in negatively influencing the negative mental states in women. This finding lends empirical weight to interventions designed to enhance both the environmental and individual strengths. However, because positive mental states was modeled (and identified) as a mediating variable in the present study, we suggest that successful interventions designed to enhance the family functioning may reasonably be expected to lead to both development of strengths, and subsequent reduced negative mental states in women. Thus, even though reduced mental distress is ultimately the desired outcome, the focus of interventions can remain on the enhancement of family functioning. One practical implication of this analysis is that we need not to attempt to direct target the negative mental states. In contrast, by remaining focused on developing the healthy family functioning, emphasizing strengths and capacities, we can expect development of positive mental states and subsequent reductions in negative mental states.

Although not explicitly tested in this study, the results of this study suggest that combined interventions targeting family functioning and increasing positive mental states may be particularly useful in addressing negative mental states in women.

\section{Limitations and recommendations:-}

The present study has several limitations. First, the sample was limited to female students enrolled in University of Kashmir. The study is also limited by fact that it did not use longitudinal data. Longitudinal data would enable an estimation of the causal effects of family functioning on mental states taking into account previous levels of each of these variables. Often when longitudinal modeling is undertaken, the size of the effects between variables is attenuated by effects within variables across time. Thus, although the structural paths in the present model were estimated in such a way as to reduce measurement error, it is nevertheless possible that the magnitude of these structural paths would be less in a longitudinal study.

Given the limitations noted above, we suggest that future studies could incorporate more diverse samples, providing longitudinal data, in order to verify the results of the study under more robust sampling and statistical conditions.

\section{Conclusion:-}

In this study we demonstrate that mental states in women can be accurately modeled as a function of family. This modeling suggests that both family functioning and positive mental states are key variables for negative mental states in women. 


\section{References:-}

1. Ackerman, N. (1959): Theory of family dynamics. Psychoanalysis and the Psychoanalytic Review, 46 (4): 3350.

2. Aspinwall, L.G., \& Taylor, S.E. (1992): Modeling cognitive adaptation: A longitudinal investigation of the impact of individual differences and coping on college adjustment and performance. Journal of Personality and Social Psychology, 63: 989-1003.

3. Bandalos, D.L., \& Finney, S.J. (2001): Item parceling issues in structural equation modeling. In Marcoulides GA, Schumacker, RE (eds) New developments and techniques in structural equation modeling: Mahawah, NJ: Lawrence Erlbaum Associates, pp. 269-296.

4. Bowen, M. (1978): Family therapy in clinical practice. New York: Jason Aronson.

5. Byrne, B. (1998): Structural equation modeling with Lisrel, Prelis, and Simplis: Basic concepts, applications, and programming. Philadelphia: Lawrence Erlbaum.

6. Byrne, B.M. (2001): Structural equation modeling with AMOS: Basic concepts, applications, and programming. Mahawah, NJ: Lawrence Erlbaum Associates.

7. Carr, A. (2004): Positive psychology: The science of happiness and human strength. New York, NY: BrunnerRoutledge.

8. Chauhan, S. (2006): The role of family environment in the development of neurotic tendencies and coping skills. Unpublished Doctoral Thesis, Himachal Pradesh University, Shimla, India.

9. Chorpita, B.P. and Barlow, D.H. (1998): The development of anxiety: The role of control in the early environment. Psychological Bulletin, 124: 3-21.

10. Cohen, J. (1988): Statistical power analysis for the behavioral sciences. San Diego: Academic Press.

11. Das, V. (1994): Moral Orientations to Suffering: Legitimation, Power and Healing. In L. Chen, A. Kleinman and N. Ware (eds) Health and Social Change in International Perspective (pp 139-170). Boston: Harvard Series on Population and International Health.

12. Epstein, N.B., Baldwin, L.M., \& Bishop, D.S. (1983): The McMaster Family Assessment Device. Journal of Marital and Family Therapy, 9(2): 171-180.

13. Epstein, N.B., Bishop, D.S., and Levin, S. (1978): The McMaster Model of Family Functioning. Journal of Marital and Family Counseling, 4: 19-31.

14. Fingerman, K., and Bermann, E. (2000): Applications of family systems theory to the study of adulthood. International Journal of Aging and Human Development, 51(1): 5-29.

15. Fornell, C.R., and Larcker, D.F. (1981). Two structural equation models with unobservable variables and measurement error. Journal of Marketing Research, 18: 39-50.

16. Friedmann, M.S., McDermut, W.H., Solomon, D.A., Ryan, C.E., Keitner, G.I., and Miller, I.W. (1997): Family functioning and mental illness: A comparison of psychiatric and nonclinical families. Family Process, 36: 357367.

17. Herth, K.H. (1992): Abbreviated instrument to measure hope: Development and psychometric evaluation. Journal of Advanced Nursing, 17: 1251-1259.

18. Hills, P. and Argyle, M. (2002): The Oxford Happiness Questionnaire: a compact scale for the measurement of psychological well-being. Personality and Individual Differences, 33: 1073-1082.

19. Hu, L., and Bentler, P.M. (1999): Cutoff criteria for fit indices in covariance structure analysis: Conventional criteria versus new alternatives. Structural Equation Modeling, 6(1): 1-55.

20. Jackson, D.D. (1965): Family rules: Marital quid pro quo. Archives of General Psychiatry, 12: 589-94.

21. Joshi, R. and Tomar, A.K. (2006): Effect of family environment on behavioural problems and family dynamics. Journal of Research and Applications in Clinical Psychology, 9: 51-56.

22. Kaplan, D. (2000): Structural equation modeling: Foundations and extensions. Newbury Park, CA: Sage.

23. Kaya, F. (1985): Symptomatic differences between the sexes in rural Mexico. Culture, Medicine and Psychiatry, 9: 27-57.

24. Keitner, G.I., Miller, I.W., and Ryan, C.E. (1993): The role of family in major depressive illness. Psychiatric Annals, 23(9): 500-507.

25. Khordzanganeh, F., Heidarie, A. R., and Naderi, F. (2014): The relationship between emotional intelligence, happiness and resiliency with mental health in male and female high school students of Ramhormoz city. Indian Journal of Fundamental and Applied Life Sciences, 4 (2): 633-641.

26. Kline, R.B. (1998): Principles and practice of structural equation modeling. New York: Guilford.

27. Little, T.D., Cunningham, W.A., Shahar, G., and Widaman, K.F. (2002): To parcel or not to parcel: Exploring the question, weighing the merits. Structural Equation Modeling, 9: 151-173. 
28. Lovibond, S.H. and Lovibond, P.F. (1995): Manual for the Depression Anxiety Stress Scales (2nd ed.). Sydney: Psychology Foundation (Available from The Psychology Foundation, Room 1005 Mathews Building, University of New South Wales, NSW 2052, Australia) http://www2.psy.unsw.edu.au/groups/dass/

29. Lu, L., and Argyle, M. (1992): Receiving and giving support: Effects on relationships and well-being. Counseling Psychology Quarterly, 5: 123-133.

30. Malik, I. A., Bukhtiari, N., and Good, M.J. D. (1992): Mothers' fear of child death: A Study in Urban and Rural Communities in Northern Punjab, Pakistan. Social Science and Medicine, 35: 1043-1053.

31. Marcia, A. (1997): The Ethics of Clinical Research in the Third World. Journal of Medicine 337(12): 847-849.

32. McCubbin, H.I., and Thompson, A.I. (eds) (1991): Family assessment inventories for research and practice. Madison: university of Wisconsin.

33. Miller, I.W., Keitner, G.I., Whisman, M.A., Ryan, C.E., Epstein, N.B., and Bishop, D.S. (1992): Depressed patients with dysfunctional families: Description and course of illness. Journal of Abnormal Psychology, 101(4): 637-646.

34. Minuchin, S. (1974): Families and family therapy. Cambridge, MA: Harvard University Press.

35. Naeem, S. (1992): Vulnerability factors for depression in Pakistani women. Journal of the Pakistan Medical Association, 6: 137-138.

36. Nancy, S.H. (1995). The primacy of the ethical: Propositions for a militant anthropology. Current Anthropology, 36(3): 409-421.

37. Nations, M. K. and Rebhun, L. (1988): Angels with wet wings won't fly: Maternal sentiment in Brazil and the image of neglect. Culture, Medicine and Psychiatry, 12:171-200.

38. Parker, G. (1983): Parental overprotection: A risk factor in psycho-social development. New York: Grune \& Stratton.

39. Rosenfield, A., and Maine, D. (1985): Maternal mortality - A neglected tragedy. Where is the "M" in MCH? The Lancet, 2: 83-85.

40. Scheier, M.F., Carver, C.S., and Bridges, M.W. (1994): Distinguishing optimism from neuroticism (and trait anxiety, self-mastery, and self-esteem): A reevaluation of the life orientation test. Journal of Personality and Social Psychology, 67: 1063-1078.

41. Schwab, J.J., Stephenson, J.J., and Ice, J.F. (1993): Evaluating family mental health: History, epidemiology, and treatment issues. New York: Plenum Press.

42. Seligman, M.E.P. (2002): Authentic happiness: Using the new positive psychology to realize your potential for learning and fulfillment. New York, NY: Free Press.

43. Seligman, M.E.P. (1998): Building human strength: Psychology's forgotten mission. Retrieved from http://www.apa.org/monitor/han98/pres.html.

44. Seligman, M.E.P., and Csikszentmihalyi, M. (2000): Positive psychology: An introduction. American Psychologist, 55: 68-78.

45. Sharma, A., Verma, R. and Malhotra, D.K. (2008): The role of pathogenic family patterns in the development of generalized anxiety in the urban and rural women. Journal of Personality and Clinical Studies, 24: 183-92.

46. Taylor, S.E., Kemeny, M.E., Reed, G.M., Bower, J.E., and Grunwald, T.L. (2000): Psychological resources, positive illusions, and health. American Psychologist, 55: 99-109.

47. Van Velsor, P., and Cox, D. (2000): Use of the collaborative drawing technique in school counseling practicum: An illustration of family systems. Counselor Education and Supervision, 40(2): 141-53.

48. Vander Kwaak, A., Vanden Engel, M., and Richters, A., (1991): Women and Health. Vena Journal, 3: 2-33.

49. Von Bertalanffy, L. (1973): General systems theory. New York: George Braziller.

50. Ware, N. and Good, M. (1995): Women. In R. Desjarlais, A. Kleinman, L. Eisenberg, B. Good (eds) World Mental Health: Problems, Priorities, and Responses in Low-Income Countries (pp 179-206). Oxford University Press.

51. Westley, W. A., and Epstein, N.B. (1969): The silent majority: Families of emotionally health college students. San Francisco: jossey-Bass.

52. Whall, A.L. (1986): Family therapy theory for nursing. Connecticut: Prentice - Hall. 